\title{
EDUCAR DESDE LA LITERATURA: ENRAIZAMIENTO CULTURAL PARA UNA PEDAGOGÍA COMPRENSIVA
}

\section{TO EDUCATE FROM THE LITERATURE: CULTURAL ENRAIZAMIENTO FOR AN UNDERSTANDING PEDAGOGY}

\section{Dr. C. Bárbara Maricely Fierro Chong ${ }^{1}$}

“... Conocer diversas literaturas es el medio mejor de libertarse de la tiranía de algunas de ellas; así como no hay manera de salvarse del riesgo de obedecer ciegamente a un sistema filosófico, sino nutrirse de todos, y ver como en todos palpita un mismo espíritu..."

José Martí

\section{RESUMEN}

La educación de las nuevas generaciones

1 https://orcid.org/0000-0002-7177-1860. Profesora Titular del Centro de Estudios Educativos de la Universidad de Matanzas. Presidenta de la Cátedra de Lectura y escritura, de la Universidad de Matanzas. fundada en 1994. Jefa del proyecto de Investigación La enseñanza aprendizaje de las lenguas y la literatura en la educación de la personalidad, Miembro de la Subcomisiòn Nacional de planes y programas del Instituto Central de Ciencias Pedagógicas de Cuba. Par Redipe, 2018. Coordinación científica de Redipe en Cuba. tiene en la literatura un contenido de infinitas posibilidades para el logro de aprendizajes autónomos, significativos, de enraizamiento cultural desde lo cognitivo -afectivo y creativo. Las investigaciones pedagógicas en el campo de la lectura y la escritura, revelan el insuficiente lugar que se otorga y aprovecha al empleo de la literatura para la comprensión del mundo y esencia edificadora. Ante tal problemática, la autora de este artículo realizó indagaciones empíricas y teóricas en el marco del proyecto de investigación que dirige. Se reveló la necesidad de una actualización de los docentes en los contenidos de la Literatura y los recursos didácticos para cumplir las aspiraciones de la educación literaria.

\section{SUMMARY}

The education of the new generations has in the literature a content of infinite possibilities for the achievement of autonomous, significant 
learnings, of cultural enraizamiento from the cognitivo - affective and creative. The pedagogic investigations in the field of the reading and the writing, reveal the insufficient place that is granted and he/she takes advantage to the employment of the literature for the understanding of the world and essence builder. Before such a problem, the author of this article carried out empiric and theoretical inquiries in the mark of the investigation project that directs. The necessity of an upgrade of the educational ones was revealed in the contents of the Literature and the didactic resources to complete the aspirations of the literary education.

PALABRAS CLAVE: educación literaria, didáctica de la literatura, pedagogía, formación, comprensión.

WORDS KEY: literary education, didactics of the literature, pedagogy, formation, understanding

\section{INTRODUCCIÓN}

Los objetivos de la Unesco (2015) para el desarrollo sostenible articulan las ingentes necesidades que reclama la especie humana y su vida en la tierra: examinar el aprendizaje desde los lentes de la educación para la sostenibilidad, dar lugar a las emociones y con ello a las artes y las humanidades, desde una mirada comprensiva y edificadora (Arboleda, $2018)^{2}$.

Educar desde literatura es una actividad edificadora y nutricia si se encamina desde el disfrute y el placer, en ella se imbrican procesos intelectivos, emocionales y volitivos, en los cuales lo emotivo tiene un lugar de preferencia, porque lo primario está en la palabra, su valor connotativo, denotativo y de expansión cultural, de enraizamiento, identidad, creatividad y actitud transformadora y ética. Ella es esencial en la

2 Arboleda, J. C. (2015). Formación para la vida: de las competencias a la comprensión edificadora. Revista Boletín Redipe, ISSN-e 2256-1536, Vol. 4, No. 12, 2015, págs. 20-25 creación de un pensamiento crítico y creativo para entender y transformar la compleja realidad y devolverla desde una mirada personal, esencia de una pedagogía edificadora.

Algunas claves para entender, proyectar y contribuir a la educación literaria remiten a que intelección y sentimientos van en unidad, no se puede separar el ejercicio de la inteligencia de los sentimientos, las emociones que generan a su vez cualidades morales y creativas. La literatura cobra valor cuando el lector hace suya otras interpretaciones, se sumerge en su universo y proyecta una reacción hacia ella: positiva o negativa, de indagación, exploración o sencillamente de búsqueda creativa.

Gabriela Mistral en Pasión de leer "Lo que no se hace pasión en la adolescencia se desmorona hacia la madurez relajada" ( $p$. 314), la sistematicidad es una clave esencial, que al decir de la Mistral; "Hacer leer, como se come, todos los días, hasta que la lectura sea, como el mirar, un ejercicio natural, pero gozoso siempre..." (ídem=.

Las indagaciones con profesores en ejercicio de la asignatura Español-Literatura en la enseñanza media (básica y superior) y metodólogos de la asignatura a diferentes niveles, revelò que en el contexto educativo actual no puede prescindirse de la literatura y se reclama activar novedosas formas que promuevan sentidos y significados personales.

En correspondencia con ello, el proceso investigativo desarrollado por la autora en el marco del proyecto de investigación responde a la necesidad de indagar qué literatura y cómo y desde qué perspectivas integrarlas en el hacer de la escuela en una década marcada por la presencia absorbente de la visualidad y diferentes lenguajes como el tecnológico y digital. 


\section{FUNDAMENTOS TEÓRICO- METODOLÓGICOS}

En las sesiones del Congreso de Lectura 2018, para leer el siglo XXI, convocado por el Comité de la IBBY, desarrollado en La Habana, se revelaron algunas ideas que incitan al pensar en torno a la lectura como nodo interdisciplinario, intercultural e intertextual que promueve el pensar crítico, reflexivo y creativo:

- Dar espacio a los niños, adolescentes y jóvenes a la lectura del mundo desde sus propios lentes, con sus matices de significación y sentido de acuerdo con sus experiencias.

- Promover una lectura desde los diferentes códigos de sentido para educar la sensibilidad, la visualidad, la emocionalidad, la interculturalidad. En este proceso ocurre una integración de la palabra dialogada, en que se manifiesta una adhesión al lector activo, en diferentes formatos y códigos, relacionada con la proyección y ejecución de talleres de lecturas, espacios de compartir lecturas, escritura creativa

- Conciliar alianzas desde todos los agentes participantes, escuela, familia, comunidad para que el niño crezca desde sus propias visiones y perspectivas en un mundo cambiante.

- Enseñar desde una ética y unos valores de la verdad, la equidad social, cultural, la no violencia.

En el proceso de investigación en el marco del proyecto científico La enseñanza aprendizaje de las lenguas y la literatura en la educación de la personalidad, de la Universidad de Matanzas, adscripto a un Programa Nacional liderado desde el Instituto Central de Ciencias Pedagógicas, Cuba, se desarrollaron tareas dirigidas a actualizar los términos y estado del arte en torno a la educación literaria como plataforma teórica de la implementación de las transformaciones educacionales que se realizan en el proceso de perfeccionamiento continuo, y en el cual la universidad, como escenario de formación desempeña un rol de liderazgo científico de acompañamiento a las instituciones educativas de los niveles precedentes.

En una búsqueda de los caminos de la literatura a la educación literaria, se comparte con Teresa Colomer que

[...] el objetivo de la educación literaria es... el de contribuir a la formación de la persona, una formación que aparece indisolublemente ligada a la construcción de la sociabilidad y realizada a través de la confrontación con textos que explicitan la forma en la que las generaciones anteriores y las contemporáneas han abordado la valoración de la actividad humana a través del lenguaje. (Colomer, T. 2005, p. 38)

Educar literariamente en esta segunda década del siglo XXI reclama en la escuela de una actitud creativa, proactiva, para "aprender y enseñar a leer literariamente otra vez", comienza por la disposición de los docentes de:

- Vivenciar y trasmitir en vivo el proceso de la creación literaria, desde sus propias experiencias lectoras mediante estrategias de comprensión que articulen lo vivencial, intelectual, empático.

- Contagiar con la lectura literaria desde todos los escenarios posibles.

- Activar las múltiples relaciones de significados y sentidos de la literatura en diversos formatos.

- Revelar la relación de valores y conocimientos, vivencias y creatividad desde las obras pertenecientes a diferentes géneros literarios.

- Educar desde la interculturalidad, la multiculturalidad y transculturalidad, para trasmitir los valores identitarios culturales que se amalgaman para conformar el gusto 
estético y el enraizamiento cultural de los niños, adolescentes y jóvenes.

\section{MÉTODOS Y RESULTADOS}

En el marco de la investigación apoyada en métodos de indagación empírica con énfasis en la observación, la entrevista a profundidad, la observación de clases con la unidad de estudio identificada por profesores en ejercicio, se realizaron acciones de diagnóstico para corroborar la hipótesis de trabajo de la necesaria actualización de los docentes en lo cognitivo, lo afectivo motivacional y lo comportamental para hacer del encuentro con la literatura un contenido que fascine y genere sentidos y significados que amplíen el universo cultural y espiritual.

En el contexto educativo cubano, el intercambio con docentes de los niveles: secundaria básica y preuniversitario, se revelaron las siguientes informaciones:

- La diversidad en la formación de los docentes que dirigen el proceso de enseñanza aprendizaje es una variable a considerar, y en la cual se muestra poca o limitada experiencias de lecturas literarias.

- La reducción de la obra literaria a condición de mero tipo de texto, aquilatado al mismo nivel que los diferentes tipos textos, sin proyectar la significación que posee de tener un particular sentido en que subyacen y entretejen múltiples significados.

- La carencia de sistematicidad e inspiración para leer literatura de factura estética, sentirla como necesidad espiritual.

- Selección de otros tipos de textos que no privilegian la lectura literaria y la

La potencialidad radica en que se observa la disposición de maestros, docentes e investigadores de repensar algunas acciones en el orden didáctico y metodológico para contribuir a la educación literaria en los ambientes escolares.

De ahí que se resolvió retomar algunas ideas referidas a la preparación del docente de manera que los pongan en situación de éxito ante la problemática de "ofrecer en vivo el proceso de la creación literaria" como expresó en una conferencia el profesor universitario, crítico literario, ensayista y comentarista de cine, Mario Rodríguez Alemán, porque la lectura literaria no es un simple adorno, sino imprescindible atributo del acto cultural y creador que es la educación:

- El trabajo metodológico, colectivo e individual y de superación de los docentes, es una fuente de experiencias que incorpora $y$ articula con las diferentes figuras del posgrado académico, para proyectar $y$ potenciar a los docentes como lectores de literatura, a generar vivencias, propiciar el interés por lo que los rodea, el amor y los valores hacia los demás, y un mayor grado de participación e implicación personal en la realidad en que vive.

- La actualización de los docentes en los saberes literarios con autores, obras, por géneros o formas genéricas emergentes en los escenarios editoriales contemporáneos, tales como el microcuento, la novela histórica, el testimonio, conducentes a descubrir lo que se dice y qué se recepciona a partir de la hibridez genérica que distingue a una parte de la producción literaria actual.

- La lectura literaria para una educación inclusiva, con énfasis en la literatura infantil y juvenil de autores cubanos contemporáneos, con el empleo de recursos didácticos.

- La importancia de retomar y particularizar en las formas genéricas con sus características y posibilidades expresivas.

La educación literaria es un proceso que coadyuva a formar lectores, que sistematiza 
el enraizamiento cultural. Asumirla en la perspectiva escolar integrada a otros saberes ya sean lingüísticos, históricos o artísticos, puede apoyarse en principios pedagógicos:

- La interdisciplinariedad: la lectura de la literatura se nutre de la relación con otras áreas del saber tales como la historia, la geografía, la filosofía, las artes, que posibilitan puntos de encuentros interdisciplinarios de carácter sociocultural, al propiciar además, la reflexión en torno a las problemáticas de la realidad, la asociación que puede establecerse con otros discursos para relacionar los contenidos enunciados y devolverlos creadoramente. La intertextualidad como procedimiento cultural, que revela al texto como unidad abierta, que renueva y provoca en su relación con otros textos, lo que Bajtín llamó un palimpsesto, es decir, un texto en que se leen las huellas de otros, en que se imbrica lo anterior y lo nuevo, fundidos en un nuevo significado

- La unidad de la comunicación y la actividad: la literatura es un acto comunicativo y se comparte en la actividad en el marco de los diferentes escenarios, lo que propicia la interacción entre pares o en grupos de manera activa para construir nuevos significados generados de la lectura literaria de forma creativa, flexible, original, hallar respuestas diversas con orientaciones valorativas que amplían el universo de experiencias y valores. La inserción de la lectura en prácticas socioculturales en correspondencia con la edad, los intereses y su formación de lectores como parte de su proyecto de vida, por la presencia del texto literario en actividades docentes, extradocentes y extracurriculares en la formación docente, sustentado en una concepción interdisciplinar.

- La unidad de lo cognitivo y lo afectivo: la literatura es propicia para el desarrollo de significados y sentidos en los estudiantes, al contribuir al conocimiento de sí y de los demás a partir de la comprensión literaria de lo que ofrece el autor y que provoca múltiples recepciones en los lectores. Lo vivencial moviliza el conocimiento, el comportamiento, la actividad creadora y la actuación transformadora hacia la realidad, en la medida en que al leer literariamente se traspasen los límites de lectura para incorporar lo literario como un proceso de particular sentido.

La unidad de lo social y lo individual: el carácter y enfoque plural e integral de la literatura y por su naturaleza intrínseca, plurisemántica, simbólica, asociativa, no se le puede describir de forma univalente, sino desde la carga de subjetividad e intersubjetividad que ello supone. Como plantea Vigotsky en "cada edad se produce de forma diferente la interrelación entre lo interno y externo". (1996, pág. ${ }^{265}$ ) La lectura literaria contribuye al desarrollo intelectual y emocional de la personalidad, en la reafirmación de cualidades creativas y valóricas se reconstruyen identidades personales y sociales. Este proceso de sistematización y síntesis, tiene carácter dialéctico dado por una actitud de reconstrucción, la lectura literaria ayuda a la definición de metas en la proyección de vida en el contexto social. La interacción con la literatura promueve orientaciones valorativas y se revela en la subjetividad del ser humano, lo que coloca también la mirada en sus posibilidades para la inclusión educativa.

- Elcaráctercientíficoeideológico delaeducación literaria: el proceso de investigación científica y de innovación con el empleo de métodos de investigación de orientación cualitativa, revela que los referentes filosóficos, psicológicos, pedagógicos, sociológicos y literarios articulan coherentemente como soporte de acciones encaminadas a en al proceso de interacción 
con la literatura en diferentes ámbitos. La literatura legitima las relaciones de las esferas diversas de la experiencia vital humana, y los estudios literarios, centrados en la lectura y disfrute del texto literario, se entrecruzan con lo que Juan R. Montaño llama los sistemas con los que se concibe esta experiencia: filosófico, histórico, social, psicológico, ético, lingüístico, entre otros.

- El uso de las Tecnologías de la Información y las Comunicaciones (TIC): su empleo va más allá de ser herramienta informática de búsquedas, y de interactuar y comprender lo que se produce en diversos códigos; sino propicia a los estudiantes ser productores de nuevos significados con el empleo de la lectura literaria digital, el desarrollo de cualidades creativas, independencia cognoscitiva, flexibilidad e imaginación como resultante de la interacción con productos informáticos o su elaboración como reconstrucción delo aprendido.

A partir de sostener esta posición que conduce a otorgar un lugar a los componentes funcionales de la clase: comprensión, análisis y construcción de significados. ¿Qué significa aprender a leer literariamente otra vez?, esta es una idea clave desde la formación del profesorado, y se inserta en el proceso de enseñanza, para desarrollar la capacidad de encontrarse con la obra literaria, no como mero texto informativo, sino desde su prístina función expresiva y de actividad creadora.

Desde la posición de receptor de la literatura, el interés didáctico radica en que los lectores pueden apreciar la obra literaria desde su interacción con otros creados en diferentes formatos y viceversa, es decir, no hay un solo carril de lectura, sino trayectos que se bifurcan y entrecruzan en la experiencia de lectura y la lectura de la experiencia. La mediación del docente es un factor importante, porque desde el diagnóstico facilita desarrollar en los estudiantes aquellas potencialidades creativas que posee, de ahí que es crucial tener un maestro que contagie, cautive, hechice con la palabra, trasmita por las venas la pasión de leer a que se refiere Gabriela Mistral.

Se retoma entonces la idea de que la literatura es ejercicio vivencial, de experiencia sociocultural, que ayuda a conectar realidades diversas, dese todas las áreas del saber la literatura es ofrecimiento, la geografía, las artes, la historia, la botánica, la zoología, las matemáticas y hasta la Física tienen resguardo y puerto seguro en la literatura.

Leer literariamente otra vez es la posibilidad de entrar en contacto mediante la literatura con otros mundos lejanos o cercanos, y la lectura a viva voz es un procedimiento del método de lectura creadora, que conecta la literatura con otros tipos de textos como artículos de opinión, noticias, biografías, viñetas, que permiten ampliar el universo de las experiencias estético - literarias, y que pueden leerse en formatos físicos o electrónicos.

Contar, narrar es parte del hechizo que puede hacer el maestro, cuando comparte con sus estudiantes en clases un pasaje narrativo que lo acerca a la historia de $\mathrm{n}$ proceso o fenomeno o hecho.

En las últimas décadas, la minificción, el cuento corto o el cuento ultracorto en Hispanoamérica es una alternativa para desarrollar la educacion literaria porque sus caracteristicas constructivas posibilitan activar experiencias y sensaciones en el lector en breve tiempo, e incentivar la curiosidad, el interés por leer y reflexionar sobre el rol individual y colectivo que poseemos en la perspectiva cultural del desarrollo humano.

En la búsqueda de procedimientos metodológicos, se puede dibujar un mapa para incluir trayectos de lecturas dirigido hacia: ¿Cómo y desde qué ideas leer determinadas 
obras de la literatura universal que conforman el canon de lecturas escolares de los programas de estudio vigentes? ¿Qué obras de la literatura infantil y juvenil y géneros emergentes como la minificcion pueden ser incorporados al proceso formativo? ¿cómo articular la lectura y construccion de nuevos significados con el empleo de las tecnologías?

El escritor uruguayo Eduardo Galeano legó a la cultura latinoamericana una visión particular de la experiencia humana, recogida en múltiples obras que hablan del hombre, su historia, sus conflictos, en su libro Espejos, una historia casi universal, Premio de narrativa "José María Arguedas", Casa de las Américas, 2014, escribió:

\section{FUNDACIÓN DE LA CONTAMINACIÓN}

Los pigmeos, que son de cuerpo corto y memoria larga, recuerdan los tiempos de antes del tiempo, cuando la tierra estaba encima del cielo.

Desde la tierra caía encima del cielo una lluvia incesante de polvo y basura, que ensuciaba la casa de los dioses y les envenenaba la comida.

Los dioses llevaban una eternidad soportando esa descarga mugrienta, cuando se les acabó la paciencia.

Enviaron un rayo que partió la tierra en dos. Y a través de la tierra abierta lanzaron hacia lo alto el Sol, la Luna y las estrellas, y por ese camino subieron ellos también. $Y$ allá arriba, lejos de nosotros, a salvo de nosotros, los dioses fundaron un nuevo reino.

Desde entonces estamos abajo. (Galeano, E. 2015. Pág. 17).

La lectura de la obra anterior ofrece al lector la posibilidad de captar el sentido o los sentidos de lo dicho y lo no dicho e imaginar significados desde la actividad lúdica que se experimenta en la interacción con el texto, provocada por las características que lo distinguen como la brevedad y concisión, de decir mucho en tan breve espacio, y economía de recursos del lenguaje.

A lo largo del tiempo, se lee y parece escrito en este minuto por lo que pueden provocar en el lector, la reflexión crítica y al mismo tiempo de carácter edificador y al mismo tiempo comprensivo del mundo en que vivimos.

La hibridez genérica, la presencia de un tema de pertinencia individual y social para la humanidad, acercan al lector joven a este texto que habla de los humanos, de nosotros para invitar al diálogo, porque la literatura en su capacidad expresiva conduce a pensar, sentir y actuar. ¿Qué percepciones y sensaciones provoca en un lector este texto? ¿Pudiera eslabonarse desde él la lectura de otros textos literarios y no literarios? ¿Cómo leerlo en el aula que incite al placer estético en un círculo de lectura como es la escuela? ¿qué debe conocer el docente para incitar a la lectura inteligente, audaz de textos como el de Eduardo Galeano?

Es un texto que provoca reflexionar sobre nosotros y los otros, una ética que subyace en las acciones humanas: la contaminación y los peligros ecológicos tienen su origen en el comportamiento humano y que está refrendado como uno de los objetivos de la Unesco.

Al tomar de referente las propuestas de trayectos de lectura de Montaño y Abello (2015), se abordó la lectura desde la diferencia, lo que ratifica las posibilidades de la literatura para el desarrollo de una pedagogía que sea edificadora de los mejores valores, potencie en los escolares su capacidad comprensiva hacia el otro y los otros.

La literatura latinoamericana es fuente de múltiples registros literarios que facilitan transitar por trayectos de lecturas como ejes transversales que activan la rica fibra emocional 
desde la experiencia de leer a viva voz, de incorporar en la lectura compartida saberes de la emocionalidad, la sensibilidad frente al otro.

La obra poética de Gabriela Mistral (Vicuña, Chile, 1889 -Nueva York, Estados Unidos, 1957), maestra, poetisa, comprometida vocación de magisterio en las difíciles condiciones de la cordillera andina es voz lírica que mantiene vigencia en las lecturas no solo infantiles, sino juveniles.

Su poema "Los que no danzan", perteneciente a su poemario Ternura, publicado en Madrid, en 1924, en el cual recrea "... a su gusto y antojo, desvariadoramente, su mundo de realidades y encantamientos" (Quezada, p. 11), del cual en sus versos octosílabos se entra "un saber contar, que es encantar, con lo cual se entra en la magia" favorece el pensar y sentir la literatura con las posibilidades de los diversos registros de la comunicación: dialógica, la multiplicidad de interrogantes que configuran la gradación de significados, sentimientos y situaciones humanas, ante realidades diversas que invitan a dialogar en torno a la inclusión:

Una niña que es inválida

dijo: _“¿Cómo danzo yo?”

Le dijimos que pusiera

a danzar su corazón...

Luego dijo la quebrada:

-“¿Cómo cantaría yo?”

Le dijimos que pusiera

a cantar su corazón...

Dijo el pobre cardo muerto:

“- ¿Cómo danzaría yo?”

Le dijimos: "- Pon al viento a volar tu corazón"

Dijo Dios desde la altura:

“- ¿Cómo bajo del azul?"

Le dijimos que bajara

a danzarnos en la luz.

Todo el valle está danzando

en un corro bajo el sol.

A quien falte se le vuelve

de cenizas el corazón. (Mistral, G, 2014, p. 72)

En las propuestas de actividades, tomando como centro la lectura expresiva, mediada y compartida entre docente, grupo, alumno se salva del olvido, se da cabida a todos "el valle está danzando/ en un corro bajo el sol". Para sentenciar en una metáfora de alto vuelo lírico que quien dance, quien no sienta la necesidad de la comunicación con el otro y la empatía, no tiene sentimientos. La lectura para pensar y sentir sobre la diferencia.

En las exigencias del perfeccionamiento de la educación cubana, la inclusión educativa requiere de un proceso de lectura mediado por el docente que enseñe a apreciar y comprender para experimentar nuevas vivencias. Al respecto, los resultados de la investigación de Quintana, Yanira (2016) revelaron la pertinencia del desarrollo de la comprensión de lectura de textos escritos en los escolares con retraso mental, por las limitaciones que presentan para expresar la esencia de los fenómenos y las relaciones que se establecen entre ellos; generalizar los aprendizajes y transferir lo aprendido de unas situaciones a otras en su vínculo con la vida cotidiana. (Quintana, Y. 2016)

Para activar sus vivencias, aportar sentido a los significados y favorecer que de forma consciente los comprendan y transfieran 
hacia otros contextos, con el empleo de textos narrativos, que producían en los escolares aclarar aspectos del argumento de la obra, caracterizar personajes, valorar las actitudes, así como exponer los sentimientos que despertó en ellos la narración por el maestro, con el fin de comprobar la comprensión, y como resultado realizar narraciones con apoyos de preguntas y láminas. (Quintana, Y. 2016, p. 48) Justamente en esa perspectiva está el desarrollo de la capacidad de "contar".

En la educación de escolares con retraso mental la adecuación del texto y el establecimiento de los niveles de ayuda necesarios para la comprensión de significados, tanto explícitos como implícitos en él, puede contribuir a la preparación para la vida cotidiana, adulta e independiente, en tanto el aprendizaje adquirido posea significación y sentido para ellos.

La amplitud, variedad y complejidad de temas abordados en la literatura constituye una problemática para el análisis, la construcción de nuevos juicios despertados en el lector (destinatario) que contribuirá a la educación permanente de un ciudadano más reflexivo, capacitado para la obtención de la savia que potencia la buena lectura literaria en el proceso ininterrumpido de la formación humana.

Una concepción de la relación universidad sistema educativo cubano, en cada provincia de Cuba es una vía para potenciar las acciones de actualización de los docentes para dejarse acompañar de la lectura literaria, que permita a los libros saltar de los anaqueles, vivir después de las ferias del libro, danzar en coro, para que ser uno de los baluartes de la pedagogía edificadora.

En el proceso investigativo, la aplicación de la innovación en el campo de la educación literaria se desarrolló en experiencias con estudiantes de pregrado y en el posgrado, con la
En los resultados del trabajo integrador $\mathrm{e}$ interdisciplinario se elaboraron propuestas que fomentan otras vías de educar desde la literatura y se constató que cuando se organiza de manera consciente, organizada, controlada y evaluada puede ser eficaz en el trabajo metodológico del profesorado y su repercusión favorable en el proceso educativo.

\section{CONCLUSIONES}

El proceso formativo de la personalidad tiene en la educación literaria un espacio de interacción de los estudiantes y docentes con la creación literaria, que evidencia la necesidad de la gestión pedagógica para la comprensión del mundo desde esta perspectiva.

Los objetivos de la Unesco referidos a la educación inclusiva, la atención al cambio climático y el enfoque de género puede ser abordados desde la educación literaria. La aplicación de formas novedosas dadas por una manera diferente de generar los aprendizajes en la unidad de lo cognitivo y lo afectivo demuestran que educar desde la literaria es un campo abierto a las reflexiones, investigaciones e innovaciones en pedagogía.

\section{REFERENCIAS BIBLIOGRÁFICAS}

Arboleda Julio César (diciembre, 2018) La función de educar desde la perspectiva comprensivo edificadora. Conferencia magistral pronunciada en el $V$ Simposio de EDUCACIÓN Y PEDAGOGÍA, Encuentro Iberoamericano de Pares Académicos, "Reflexiones, investigaciones, programas, modelos, enfoques, perspectivas, estrategias y metodologías", Universidad de Matanzas, Cuba. 
Arboleda, J. C. (2015). Formación para la vida: de las competencias a la comprensión edificadora. Revista Boletín Redipe, ISSN-e 2256-1536, Vol. $4, \mathrm{~N}^{\circ} .12,2015$, págs. $20-25$

Ballester, Josef (1998) "Las teorías literarias y su aplicación didáctica". pp. 297-322. En: Mendoza Fillola, A., Conceptos clave en Didáctica de la Lengua y la Literatura. Barcelona: Horsori.

Bombini, Gustavo (2001) "La literatura en la escuela". Pp 1-12. En: Entre líneas. Teorías y enfoques en la enseñanza de la escritura, la gramática y la literatura. Alvarado, Maite (coord.) Flacso/Manantial. Bs. Aires

Bombini, Gustavo (2006). "Reinventar la enseñanza de la lengua y la literatura". Buenos Aires: Libros del Zorzal.

Colomer, Teresa (2001) "La enseñanza de la literatura como construcción de sentido". Pp 2-19. En: Lectura y Vida. Revista Latinoamericana de Lectura, a. 22, n. 4, Disponible en https://bit.ly/2upiOMK, (consultado febrero de 2019).

Colomer, Teresa (2005) "Andar entre libros". FCE, México.

Federación de gremios de editores de España (2016) Informe de Lectura en España. Millán, J. A. (compilador) Ministerio de Educación, Cultura y Deporte. España.

Fierro, Bárbara (2015) "Literatura e identidad, vasos comunicantes contra la desmemoria". En: Revista Amauta. Universidad del Atlántico. Barranquilla (Col.), no. 25, pp. 39-49 Recuperado en: http://investigaciones.uniatlantico.edu.co.

Fierro, Bárbara (2016). La identidad nacional y la educación en valores: vasos comunicantes desde la literatura, pp.
94-128. En: La formación de valores en Nuestra América. Colección Educación Emancipadora. Universidad Bolivariana de Venezuela, Caracas.

Fierro, Bárbara \& Díaz, Lourdes (2018) "La educación literaria o el prisma complejo con que se nos devuelve el mundo". Revista Atenas, Vol. 1. Nro. 42, disponible en: http://atenas.reduniv.edu.cu

Fierro, B \& Mañalich, R. (2012) La literatura, aprendizaje y disfrute. Editorial Pueblo y Educación, La Habana.

Galeano, Eduardo (2015) Espejos, una historia casi universal. Fondo Editorial Casa de las Américas, La Habana.

García Alzola, Ernesto (2000) Lengua y literatura. Editorial Pueblo y Educación, La Habana, 2da edición corregida 1992, 1era reimpresión.

Garriga, Ezequiel (2013) "La enseñanza de la Literatura” pág 66-122. En Didáctica de la lengua española y la literatura. Comp. Angelina Roméu, Editorial Pueblo y Educación, La Habana.

Hernández, José Emilio (2016) La inventiva simbólica de lo complejo: sobre la poesía de Roberto Manzano. Ácana, Camagüey.

Herrera, Ramón Luis (2007) La poesía y su enseñanza. Esbozo de un mapa para recorrer el laberinto Pág. 94 -140. En: La enseñanza del análisis literario: Una mirada plural. Compilación Rosario Mañalich. Editorial Pueblo y Educación. La Habana,.

Herrera, Ramón Luis (2018) Imantación de Eros y hazaña de Heracles: enseñar y aprender literatura". En revista: Temas 91 -92, La Habana. 
Lomas, Carlos (abril, 1995) "El laberinto de la literatura". En: Revista Textos de Didáctica de la Lengua y la literatura, no. 44, Monografía, dedicado a la educación literaria. Barcelona: Graó.

Mañalich, Rosario (2007) El maestro Herminio Almendros y la lectura explicada" pág. 3 14. En La enseñanza del análisis literario: Una mirada plural. (Comp. Rosario Mañalich). Editorial Pueblo y Educación. La Habana.

Mañalich, Rosario \& Fierro, B (2013) La literatura en la enseñanza media: algunas consideraciones. Pág. 144-175. En Didáctica de la lengua española y la literatura. Tomo II. Comp. Angelina Roméu, Editorial Pueblo y Educación, La Habana,.

Marinello, Juan (1961). Mesa Redonda sobre la enseñanza de la literatura. En: Suárez, A. Obras Juan Marinello. Cuba: Cultura.

Martí, José. (1992).Obras Escogidas en tres tomos. Colección Textos Martianos. Editorial de Ciencias Sociales. La Habana,

Martínez, Z. \& Murillo, Á. R. (2013)“Concepciones de la Didáctica de Literatura en Colombia en los últimos diez años". pp. 175-194. En Revista Grafía, Vol. 10(1).

Mendoza Fillola Antonio. (2008) "La educación literaria: bases para la formación de la competencia lecto-literaria. Alicante", Biblioteca Virtual Miguel de Cervantes.

Mistral, Gabriela (2014). "Los que no danzan". Pág. 72-73. En Antología de Poesía y Prosa. Selección y prólogo Jaime Quezada. Fondo de Cultura Económica, Santiago, Chile, Cuarta reimpresión,
Montaño, Juan Ramón (2009). Temas de actualización literaria. La Habana, Pueblo y Educación.

Montaño, Juan R., (2013) "Problemas actuales de la literatura". Pág. 176-187. En: Didáctica de la lengua española y la literatura. (comp. Angelina Roméu). T II. Pueblo y Educación. La Habana.

Montaño, J. R. \& Abello, A. M. (2015) "Leer y escribir. ¡Tarea de todos!". Pueblo y Educación, La Habana.

Morín, Edgar \& Delgado, C. J. (2017) "Reinventar la educación. Abrir los caminos hacia la metamorfosis". Universidad de la Habana, La Habana.

Quintana, Yanira (2016). La comprensión de textos escritos en el tercer ciclo de la educación de escolares con retraso mental, Tesis en opción al grado científico de doctor en ciencias pedagógicas. Universidad de Ciencias Pedagógicas "Enrique José Varona”, enero de

Rodríguez, Leticia. (2015) "Lo que los libros traen". Academia, La Habana,

Roni, C. \& Carlino, P. (noviembre, 2014). Leer y escribir para aprender en la escuela secundaria. Una revisión bibliográfica. VI Congreso Internacional de Investigación y Práctica Profesional en Psicología. Facultad de Psicología de la Universidad de Buenos Aires.

Saramago, José (2005) Prólogo a Don Quijote de la Mancha, Antología anotada. Pág. 22 Editorial Santillana, Venezuela, 
EDUCAR DESDE LA LITERATURA: ENRAIZAMIENTO CULTURAL PARA UNA PEDAGOGIA COMPRENSIVA

Trujillo, Francisca (2012) Entres saberes y sentires acerca de textos literarios en aulas de secundaria. México, Editorial del Magisterio "Benito Juárez" del Sindicato Nacional de Trabajadores de la Educación (S.N.T.E.),

Trujillo, Francisca (2018) La didáctica de la literatura en secundaria. Amauta, 16(32), 49-68. http://dx.doi.org/10.15648/ am(consultado en diciembre de 2018).

Vigostky, Lev S. (1996) Obras Completas. Tomo IV. Aprendizaje visor.

Villanueva, Darío (2016) Leer literatura, hoy y siempre, pp. 27-37. En: Informe de Lectura en España, 2017. Millán, J. A. (compilador) Ministerio de Educación, Cultura y Deporte. España.

Zavala, Lauro. (Enero de 2019). Minificción Contemporánea. La Ficción Ultracorta y la Literatura Posmoderna. Material de curso. Recuperado http://www.redmini. net/pdf/cursozavala.pdf . 